\title{
Targeted next-generation sequencing expands the spectrum of mitochondrial disorders
}

\author{
Si Houn Hahn*
}

\begin{abstract}
Next-generation sequencing has become a powerful tool for testing genetically and clinically heterogeneous conditions such as mitochondrial disorders. A recent study published in Science Translational Medicine underscores the considerable clinical benefits of targeted next-generation sequencing for the diagnosis of mitochondrial disorders. The findings also suggest that the genetic heterogeneity that can result in mitochondrial disease appears much broader than previously thought.

Keywords Heterogeneity, mitochondria, mitochondrial disorders, mutations, next-generation sequencing.
\end{abstract}

\section{Challenges in the diagnosis of mitochondrial disorders}

Mitochondrial diseases are thought to be the most common form of metabolic disease occurring in childhood, with an estimated frequency of 1 in 5,000 live births [1]. Because of dual genetic control, mitochondrial disorders can originate from mutations either in mitochondrial DNA (mtDNA) or, most frequently, in nuclear genes that encode mitochondrial proteins. Mitochondrial encephalopathy and stroke-like episodes (MELAS) or Leber hereditary optic neuropathy (LHON) are classical mitochondrial disorders with characteristic phenotypes. Although these diseases are defined as classical, clinical features can nonetheless be diverse, giving rise to atypical presentations and/or onset. A hallmark of mitochondrial disorders is that patients can present with any symptoms, in any organs, at any age and with any mode of inheritance. We note that increasing numbers of patients are referred to the clinic with unexplained symptoms and signs but variability in clinical presentation, which makes the diagnosis of mitochondrial diseases challenging.

*Correspondence: sihahn@uw.edu

Department of Pediatrics, Division of Genetic Medicine, University of Washington School of Medicine, Seattle Children's Hospital, Seattle, WA 98105, USA
The current diagnosis of mitochondrial disorders relies heavily on the demonstration of mitochondrial respiratory chain complex (RCC) enzyme deficiency. Despite the best efforts of many established researchers, considerable differences persist between clinical laboratories in the character, concentration and composition of substrates employed for their RCC assays, as well as the subsequent interpretation of their results. To obtain a sufficiently large sample for the assay, an invasive procedure such as muscle biopsy is often required. Muscle tissue samples are sensitive to temperature changes and prone to spurious results because of mishandling, which can impact on the accuracy of diagnosis [1]. Inconclusive or equivocal results can be distressing for the patient, not least because the current diagnostic process is lengthy, and associated with significant risks and costs.

So far, over 170 nuclear genes have been identified as causative for mitochondrial disorders presenting as neuropathy, myopathy or liver disease [2]. A recent review of 77 nuclear genes involved in mitochondrial disease, identified largely through family pedigree analysis, anticipated that approximately 10 new disease genes will be discovered each year for mitochondrial disorders [3,4]. Since approximately 1,500 proteins are likely to be involved in mitochondrial structure and function [5], many disease-causing mutations remain unidentified.

In a recent article published in Science Translational Medicine, Calvo and colleagues [6] used targeted nextgeneration sequencing (NGS) to achieve a molecular diagnosis for patients for whom a diagnosis was not previously available. Their study provides important insights into the genetic complexity underlying mitochondrial disease.

\section{Diagnosing mitochondrial disease using exome sequencing}

In recent years, NGS of the entire coding region of the genome (the exome) has been successfully deployed for the discovery of the causative genes in several Mendelian disorders [7]. These studies were performed on large families with affected individuals or on individuals with closely related clinical phenotypes. Because of the ability to sequence entire genes, whole-exome sequencing is an 
attractive choice for diagnostic testing, yet the tremendous number of variations that could potentially be discovered in genes with unknown relationships to mitochondrial function could make the interpretation of variants difficult. Also, it has been argued that the usefulness of whole-exome sequencing-based diagnostics in a clinical scenario may be limited when the family history of disease or the mode of inheritance is uncertain.

Instead of sequencing the whole exome, Calvo and colleagues [6] applied targeted exome NGS or 'MitoExome' to capture and sequence the entire mitochondrial genome and the exons of 1,034 nuclear genes encoding proteins known to be in the human mitochondrial proteome of 42 unrelated patients with suspected mitochondrial disorders. Using this approach, a high level of sensitivity and specificity of variant detection were achieved. For the efficient detection of causal alleles from the thousands of variants present in each patient, they focused on rare nuclear variants with an allelic frequency of $<0.5 \%$, and predicted to be protein modifying. They also included as causal all variants that were associated with disease in the Human Gene Mutation Database. While they observed a 5.5-fold enrichment of genes containing two prioritized alleles in patients compared with controls, only a 1.1-fold enrichment was shown for genes containing a single rare, protein-modifying variant compared with controls. As a result of this background rate, which makes it extremely difficult to positively identify dominant-acting alleles, they prioritized their analysis to only those variants consistent with a recessive mode of pathogenesis. This highlights the difficulty of unequivocally labeling variants present in candidate genes as pathogenic if they have not previously been linked to human disease. Overall, they were able to find the underlying genetic defect in 23 of 42 patients (55\%) analyzed. Ten patients carried mutations in known disease-causing genes (including one mtDNA deletion and nine nuclear genes) while the remaining thirteen patients had variants in candidate genes not previously reported in human disease; these genes included the $A G K$ (acylglycerol kinase) gene, which is thought to be involved in lipid metabolism. This finding highlights the locus heterogeneity of mitochondrial disease and broadens the diagnostic spectrum of mitochondrial disorders, suggesting that only sequencing nuclear genes for RCC subunits and assembly factors cannot always provide an accurate diagnosis for patients with suspected mitochondrial disorders. Moreover, for those patients with variants in known genes in this study, the clinical presentation alone was not sufficiently specific to allow clinicians to select and prioritize genes to be sequenced. Sequencing all candidate genes is currently not feasible with traditional sequencing methods because of the associated cost and time. Targeted NGS therefore can serve as a very useful prototype for extending the clinical evaluation in a clinically heterogeneous patient population.

\section{Challenges in variation detection and interpretation}

One of the major challenges in NGS is distinguishing which of the many sequence variants in an individual are truly deleterious. To extract useful information from NGS data, the pathogenic effects of the detected mutations, especially missense mutations, should be functionally validated. Using a lentiviral expression system, Calvo and colleagues [6] performed complementation experiments to establish pathogenicity of a mutation in NDUFB3 (NADH dehydrogenase (ubiquinone) 1 beta subcomplex, 3) carried by a patient with a complex I deficiency. Although this highlights the importance of developing high-throughput model systems for functional validation, a recent report [8] points out that NGS may fail to detect disease-associated variation, such as complex rearrangements or branch-site mutations that may not be present in the targeted region. These variants can be buried within intronic DNA, or may initially be filtered out as benign. As an explanation for the patients for whom Calvo et al. [6] failed to detect any causative mutations, they proposed that the genes were probably not in the list of 1,034 genes encoding the mitochondrial proteome, although they mention that this is less likely, as $94 \%$ of causal genes in the list encode mitochondrial proteins.

We previously explored the clinical use of targeted NGS for mitochondrial disorders involving genes that encode proteins that do not reside within the mitochondrion [9]. We analyzed 26 patients, with known or suspected mitochondrial disorders, by targeted NGS of 908 nuclear genes. Interestingly, we found that some of these patients with suspected mitochondrial disease carried mutations in genes known to encode proteins that do not reside within the mitochondrion (for example, $U B E 3 A$, which is known to be associated with Angelman syndrome, or $S C N 1 A$, a gene related to seizure disorder). Despite their clinical presentations, which were indicative of mitochondrial disorders, together with clear deficiencies in mitochondrial RCC enzymes, the RCC enzyme deficiencies appeared secondary to molecular defects in the non-mitochondrial proteins. We did not find any causative mutations in more than half of our patients, similar to the recent study by Calvo and colleagues [6]. It is therefore likely that the culprit genes are not yet included in our panel of 908 nuclear genes analyzed, supporting the idea that the genetic heterogeneity and clinical spectrum of mitochondrial disease could be much broader than is currently thought. This suggests that many individuals with mitochondrial disease remain undiagnosed and may not be receiving proper treatment. 


\section{Future of NGS as a clinical tool for mitochondrial disorders}

The recent study [6] highlights the great promise of NGS as a powerful clinical tool to diagnose complex mitochondrial disorders and perhaps other metabolic diseases. Currently, many mitochondrial disease patients are treated with vitamin cocktails, including high doses of antioxidants (vitamin $\mathrm{E}$ and $\mathrm{C}$ ), alpha-lipoic acid, CoQ10, creatine and L-carnitine. Clinical trials have been difficult to implement because of the inherent genetic variability of patients diagnosed with mitochondrial disease. As a result, the benefits of these treatments are often unclear or inconsistent. Knowing the specific molecular defects involved will help to guide the development of novel and more effective therapeutic interventions.

In addition, the cost and technical limitations of our current methodologies underscore the need for new approaches, such as targeted NGS, for the diagnosis of mitochondrial disorders. Technical advancements in NGS will continue to drive down the cost, and will help reduce the need for potentially risky, invasive muscle biopsy. Ultimately, future cost reduction will enable whole exome sequencing for patients with mitochondrial disorders together with their parents.

As pointed out by Calvo and colleagues [6], there is a clear need for clinical standards for the interpretation of genetic variants detected by NGS. The current guidelines from the American College of Medical Genetics are restricted to gene loci with an established role in disease. There are also a substantial number of non-significant variants possibly misannotated in the literature as pathogenic. Before NGS can be successfully integrated into the clinic, the currently available human mutation databases need to be carefully re-evaluated [10].

\section{Abbreviations}

mtDNA, mitochondrial DNA; NGS, next-generation sequencing; RCC, respiratory chain complex.
Competing interests

The author declares that he has no competing interests.

\section{Acknowledgements}

I thank Dr Sedensky and Dr Vasta at Seattle Children's Hospital Research

Institute for their critical comments and edits.

Published: 23 March 2012

\section{References}

1. Chen X, Thorburn DR, Wong LJ, Vladutiu GD, Haas RH, Le T, Hoppel C, Sedensky M, Morgan P, and Hahn SH: Quality improvement of mitochondrial respiratory chain complex enzyme assays using Caenorhabditis elegans. Genet Med 2011, 13:794-799.

2. Scharfe C, Lu HH, Neuenburg JK, Allen EA, Li GC, KlopstockT, Cowan TM, Enns GM, Davis RW: Mapping gene associations in human mitochondria using clinical disease phenotypes. PLoS Comput Biol 2009, 5:e1000374.

3. Tucker EJ, Compton AG, Thorburn DR: Recent advances in the genetics of mitochondrial encephalopathies. Curr Neurol Neurosci Rep 2010, 10:277-285.

4. Nouws J, Nijtmans LG, Smeitink JA, Vogel RO: Assembly factors as a new class of disease genes for mitochondrial complex I deficiency: cause, pathology and treatment options. Brain 2012, 135:12-22.

5. Calvo SE, Mootha VK: The mitochondrial proteome and human disease. Annu Rev Genomics Hum Genet 2010, 11:25-44.

6. Calvo SE, Compton AG, Hershman SG, Lim SC, Lieber DS, Tucker EJ, Laskowski A, Garone C, Liu S, Jaffe DB, Christodoulou J, Fletcher JM, Bruno DL, Goldblatt J, Dimauro S, Thorburn DR, Mootha VK: Molecular diagnosis of infantile mitochondrial disease with targeted next-generation sequencing. Sci Transl Med 2012, 4:118ra10.

7. Ng SB, Nickerson DA, Bamshad MJ, Shendure J: Massively parallel sequencing and rare disease. Hum Mol Genet 2010, 19:R119-124.

8. Tucker EJ, Mimaki M, Compton AG, McKenzie M, Ryan MT, Thorburn DR: Next-generation sequencing in molecular diagnosis: NUBPL mutations highlight the challenges of variant detection and interpretation. Hum Mutat 2012, 33:411-418.

9. Vasta V, Ng S, Turner E, Shendure J, Hahn S: Next generation sequence analysis for mitochondrial disorders. Genome Med 2009, 1:100.

10. Bell CJ, Dinwiddie DL, Miller NA, Hateley SL, Ganusova EE, Mudge J, Langley RJ, Zhang L, Lee CC, Schilkey FD, Sheth V, Woodward JE, Peckham HE, Schroth GP, Kim RW, Kingsmore SF: Carrier testing for severe childhood recessive diseases by next-generation sequencing. Sci Trans/ Med 2011, 3:65ra4.

doi:10.1186/gm321

Cite this article as: Hahn SH: Targeted next-generation sequencing expands the spectrum of mitochondrial disorders. Genome Medicine 2012, $4: 22$ 